\title{
Impact of Isoorientin on Metabolic Activity and Lipid Accumulation in Differentiated Adipocytes
}

\author{
Khanyisani Ziqubu ${ }^{1,2}$, Christo J. F. Muller ${ }^{1,2,3}$, Phiwayinkosi V. Dludla 1,4(1), \\ Sinenhlanhla X. H. Mthembu 1,2, Nnini Obonye 1,3, Johan Louw ${ }^{1,2}$, Abidemi P. Kappo 2,5(D, \\ Sonia Silvestri ${ }^{4}$, Patrick Orlando ${ }^{4}$, Luca Tiano ${ }^{4(D)}$ and Sithandiwe E. Mazibuko-Mbeje ${ }^{1,3,6, *(D)}$ \\ 1 Biomedical Research and Innovation Platform, South African Medical Research Council, \\ Tygerberg 7505, South Africa; khanyisani.ziqubu@mrc.ac.za (K.Z.); christo.muller@mrc.ac.za (C.J.F.M.); \\ pdludla@mrc.ac.za (P.V.D.); sinenhlanhla.mthembu@mrc.ac.za (S.X.H.M.); nnini.obonye@mrc.ac.za (N.O.); \\ johan.louw@mrc.ac.za (J.L.) \\ 2 Department of Biochemistry and Microbiology, University of Zululand, KwaDlangezwa 3886, South Africa; \\ akappo@uj.ac.za \\ 3 Division of Medical Physiology, Faculty of Health Sciences, Stellenbosch University, \\ Tygerberg 7505, South Africa \\ 4 Department of Life and Environmental Sciences, Polytechnic University of Marche, 60131 Ancona, Italy; \\ s.silvestri@univpm.it (S.S.); p.orlando@univpm.it (P.O.); 1.tiano@univpm.it (L.T.) \\ 5 Department of Biochemistry, Faculty of Science, University of Johannesburg, Kingsway Campus, \\ Auckland Park 2006, South Africa \\ 6 Department of Biochemistry, Faculty of Natural and Agricultural Sciences, North West University, \\ Mafikeng Campus, Mmabatho 2735, South Africa \\ * Correspondence: sithandiwe.mazibukombeje@gmail.com; Tel.: +2721-938-0341
}

Received: 1 March 2020; Accepted: 1 April 2020; Published: 13 April 2020

\begin{abstract}
The current study explored the effect of isoorientin on the metabolic activity and lipid accumulation in fully differentiated 3T3-L1 adipocytes. To achieve this, the 3T3-L1 pre-adipocytes were differentiated for eight days and treated with various concentrations of isoorientin $(0.1-100 \mu \mathrm{M})$ for four hours. Subsequently, the metabolic activity, lipid accumulation, and mitochondrial respiration were assessed. Furthermore, to unravel the molecular mechanisms that might elucidate the bioactivity of isoorientin, protein expression of the genes involved in insulin signaling and energy expenditure, such as AKT and AMPK, were investigated. The results showed that isoorientin, at different doses, could block lipid storage and enhance glycerol release, with a concomitant improvement of the metabolic activity and mitochondrial function. Although the observed beneficial effects of isoorientin on these cultured 3T3-L1 adipocytes were not consistent at all concentrations, it was clear that doses between 1 and $10 \mu \mathrm{M}$ were most effective compared to the untreated control. Moreover, the activity of isoorientin was comparable to tested positive controls of CL-316,2431, isoproterenol, insulin, and metformin. Mechanistically, protein expression of AKT and AMPK, was enhanced with isoorientin exposure, suggesting their partial role in modulating lipid metabolism and mitochondrial biogenesis. Indeed, our results showed that isoorientin has the ability to enhance mitochondrial respiration, as we observed an increase in the ATP and oxygen consumption rate. Therefore, we concluded that isoorientin has a potential to impact mitochondrial activity, lipid metabolism and energy expenditure using an in vitro experimental model of obesity.
\end{abstract}

Keywords: isoorientin; obesity; metabolic activity; lipid accumulation; 3T3-L1 adipocytes 


\section{Introduction}

Natural products are considered a safe approach in treating metabolic syndrome [1], and their therapeutic potential against obesity has been extensively reviewed [2-4]. Of interest is isoorientin, a C-glycosyl flavonoid, which is found in rooibos, among other plants, and which displays strong ameliorative effects on obesity-linked complications [5,6]. Isoorientin, also referred to as homoorientin, is a C-glucosyl flavone that was first isolated by Koeppen and colleagues in 1962 from Aspalathus linearis, a South African indigenous plant, which is widely consumed as herbal tea (rooibos) $[7,8]$. Of general interest, is evidence showing that plant extracts rich in this glucosyl flavone display enhanced anti-obesity properties, including amelioration of various metabolic complications [9-11]. Several studies conducted over the past few years in our laboratory, consistently advocate the ameliorative effect of the rooibos extracts containing isoorientin, against various metabolic disorders in different experimental models [12-14]. For example, our previous study [13] showed that an extract of fermented rooibos could block adipogenesis and affect adipocyte metabolism in cultured 3T3-L1 adipocytes. This is especially important, since although the Food and Drug Administration (FDA) approved drugs for obesity, such as orlistat and others, are effective, a major concern exists regarding their long-term use and associated adverse side effects [15]. Such limitations have raised interest in expanding research into the use of natural products as therapeutic agents to prevent obesity or its associated complications. Although some studies conducted over the years showed isoorientin, or plants rich in this natural compound, can ameliorate obesity-related complications, such as oxidative stress, inflammation, hyperlipidemia, and insulin resistance [5,16-20], there is still relatively little information about its impact as a pure compound on obesity.

In this study, we investigated a concentration-dependent effect of isoorientin on metabolic activity and lipid accumulation in fully differentiated 3T3-L1 adipocytes. We further explored some of the mechanisms involved in this process, especially those implicated in insulin signaling and energy metabolism.

\section{Results}

\subsection{Isoorientin Enhanced ATP Production and Glucose Uptake without Inducing Any Cytotoxicity in Matured 3T3-L1 Adipocytes}

The ATP assay was used to assess metabolic activity of cells after exposure to isoorientin. The results showed that that both controls (CL and Isopr) enhanced ATP content by 9\% $(p<0.0001)$ (Figure 1a). Consistently, isoorientin at 1 and $10 \mu \mathrm{M}$, enhanced ATP content by 5\% and 8\% $(p<0.05$ and $p<0.0001$ ) respectively, thereby enhancing metabolic activity of cells (Figure 1a). Interestingly, all the doses of isoorientin tested did not show cytotoxicity, as there was no significant decrease in ATP production when compared to the control.

In terms of glucose metabolism, the two antidiabetic agents, insulin $(1 \mu \mathrm{M})$ and metformin $(1 \mu \mathrm{M})$, showed enhanced effects to promote glucose uptake by $73 \%$ and $99 \%(p<0.0001)$, respectively, when compared to the control. All tested concentrations of isoorientin also significantly increased glucose uptake when compared to the control (Figure 1b). Interestingly, the positive effects of isoorientin were not dose-dependent, with the concentration of $10 \mu \mathrm{M}$ showing more potency, and a more than $52 \%$ $(p<0.001)$ improvement in glucose uptake, when compared to the experimental control (Figure 1b). 


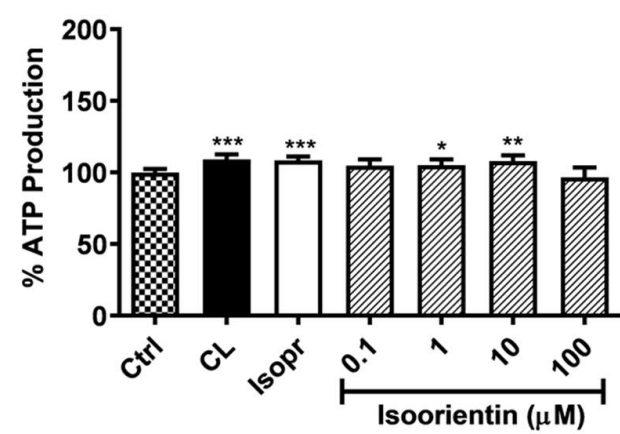

(a)

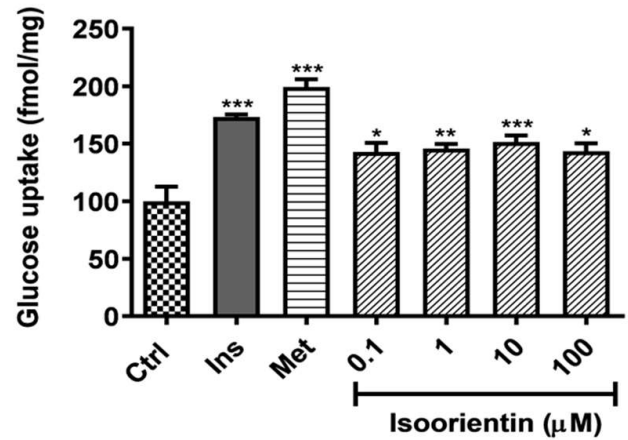

(b)

Figure 1. Isoorientin increased ATP production (a) and glucose uptake (b) in fully differentiated 3T3-L1 adipocytes. Mature 3T3-L1 adipocytes were treated with or without positive control CL-316,243 (CL), isoproterenol (Isopr), insulin (Ins), metformin (Met) used at $1 \mu \mathrm{M}$ and various doses of isoorientin (0.1, $1,10$, and $100 \mu \mathrm{M})$ for 4 hours. Results are expressed as mean \pm SEM of three independent experiments. ${ }^{*} p<0.05,{ }^{* *} p<0.01,{ }^{* * *} p<0.001$ versus normal control (Ctrl).

\subsection{Isoorientin Reduced Intracellular Lipid Accumulation and Enhanced Lipolysis in Matured} 3T3-L1 Adipocytes

The quantification and analysis of ORO was performed to determine lipid accumulation in matured 3T3-L1 adipocytes. The positive controls, CL and Isopr, had no noticeable effect after four hours of culture, while isoorientin reduced lipid accumulation, with a significant reduction of $14 \%$ at both 0.1 and $1 \mu \mathrm{M}(p<0.05)$ respectively (Figure $2 \mathrm{a})$. The reduction in lipid accumulation was accompanied by increased glycerol release from the dose of $1 \mu \mathrm{M}$, which is the end product for lipolysis (Figure $2 \mathrm{~b}$ ). However, only the isoorientin dose of $10 \mu \mathrm{M}$ significantly reduced cellular lipid content $(p<0.05)$.

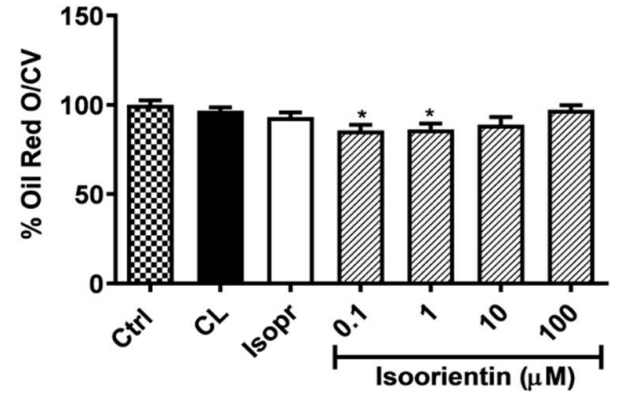

(a)

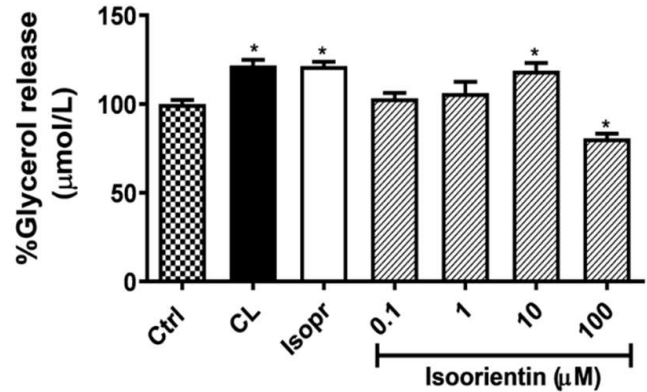

(b)

Figure 2. Isoorientin reduced lipid accumulation (a) and increased glycerol release (b) in 3T3-L1 adipocytes. Matured 3T3-L1 adipocytes were treated with or without positive CL-316,243 (CL), isoproterenol (Isopr) used at $1 \mu \mathrm{M}$ and various doses of isoorientin $(0.1,1,10$, and $100 \mu \mathrm{M}$ ) for 4 hours; subsequently, lipid accumulation was measured with Oil Red $\mathrm{O}$ and confirmed with glycerol release assay. Results are expressed as mean \pm SEM of three independent experiments. ${ }^{*} p<0.05$ versus normal control (Ctrl).

\subsection{Isoorientin Improved Mitochondrial Respiration in Matured 3T3-L1 Adipocytes}

The ability of isoorientin to enhance mitochondrial respiration in matured 3T3-L1 adipocytes was determined with the Seahorse analyzer, and the representative OCR plot is displayed in Figure $3 a$. The results showed that in terms of OCR, isoorientin increased the maximal respiration rate in all concentrations tested, with significance observed at doses of 0.1 and $1 \mu \mathrm{M}(p<0.5)$ (Figure 3b). After an 
oligomycin injection, positive controls CL and metformin, significantly enhanced ATP production by $32 \%$ and $62 \%(p<0.5$ and $p<0.001)$, respectively. All concentrations of isoorientin tested were able to increase ATP production, except for the highest dose $(100 \mu \mathrm{M})$ tested (Figure 3c).

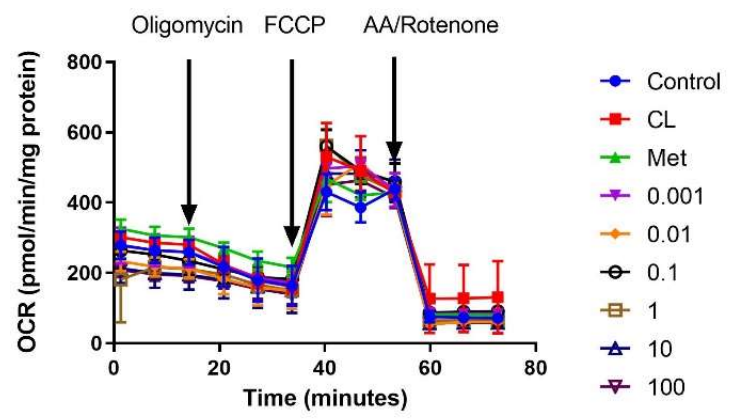

(a)

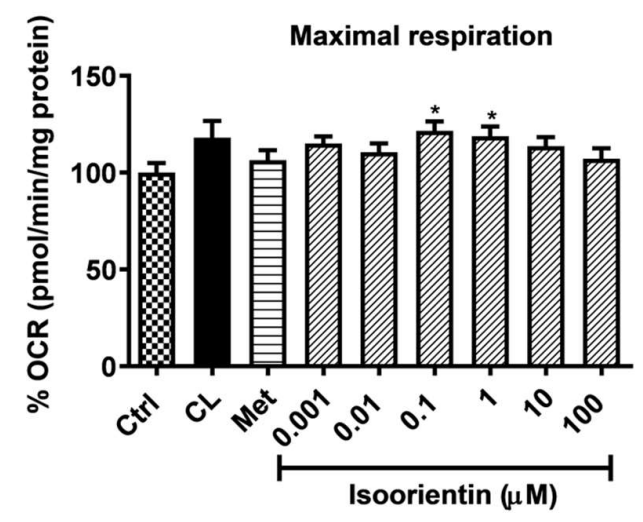

(b)

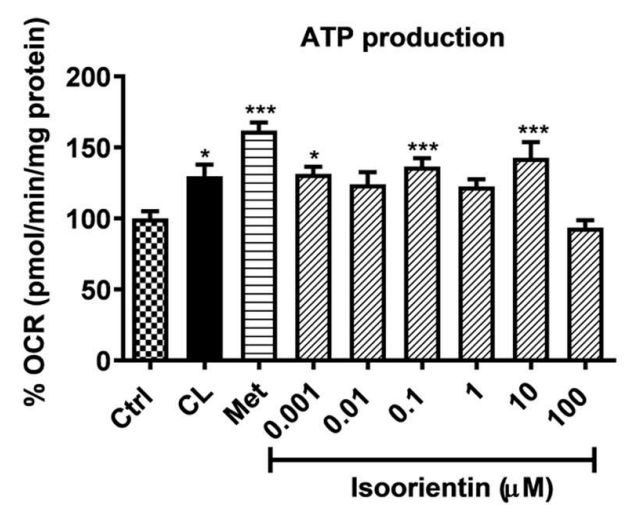

(c)

Figure 3. Isoorientin enhances mitochondrial respiration in fully differentiated 3T3-L1 adipocytes, (a) representative oxygen consumption rate (OCR) plot; (b) maximal respiration; and (c) ATP production. Matured 3T3-L1 adipocytes were treated with or without positive control CL-316,243 (CL 1), isoproterenol (Isopr ) used at $1 \mu \mathrm{M}$ and various doses of isoorientin $(0.001,0.01,0.1,1,10$, and $100 \mu \mathrm{M}$ ) for $4 \mathrm{~h}$; thereafter, mitochondrial respiration was measured using a Seahorse XF analyzer. Results are expressed as mean \pm SEM of three independent experiments. ${ }^{*} p<0.05,{ }^{* * *} p<0.001$ versus normal control (Ctrl). The numbers $0.001,0.01,0,1,1,10$, and 100 represent the isoorientin concentration used in $\mu \mathrm{M}$ units.

\subsection{Isoorientin Increased AKT and AMPK Activation in Fully Differentiated 3T3-L1 Adipocytes}

It is increasingly acknowledged that AKT and AMPK pathways are well studied signaling mechanisms, associated with the regulation of lipid metabolism, which also impact mitochondrial function. The results showed that isoorientin doses $\geq 1 \mu \mathrm{M}$, strongly enhanced AKT phosphorylation ( $p<0.001$ ), by $86 \%, 123 \%$, and $142 \%$, respectively for 1,10 , and $100 \mu \mathrm{M}$, when compared to the untreated control (Figure 4a). In addition, isoorientin at a dose of $10 \mu \mathrm{M}$, significantly enhanced the phosphorylation of AMPK, at a level comparable to that of a positive CL control $(22 \%, p<0.05)$ (Figure 4b). 


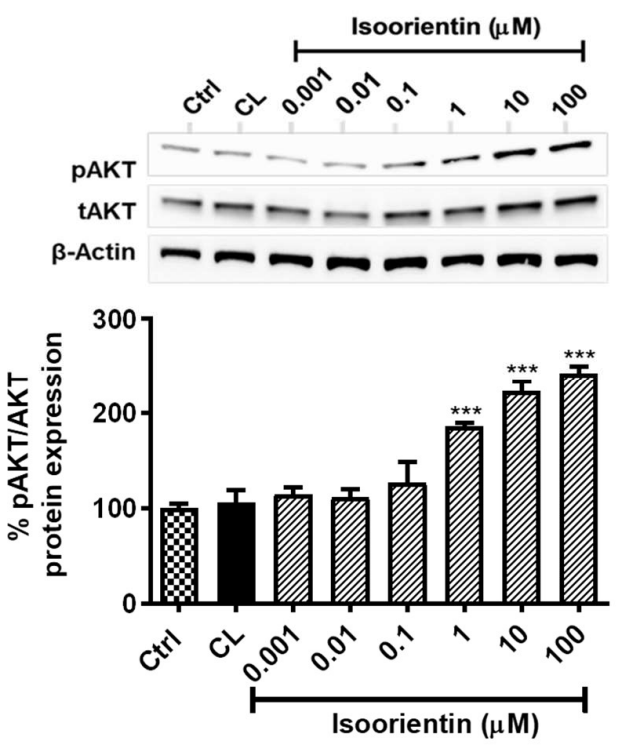

(a)

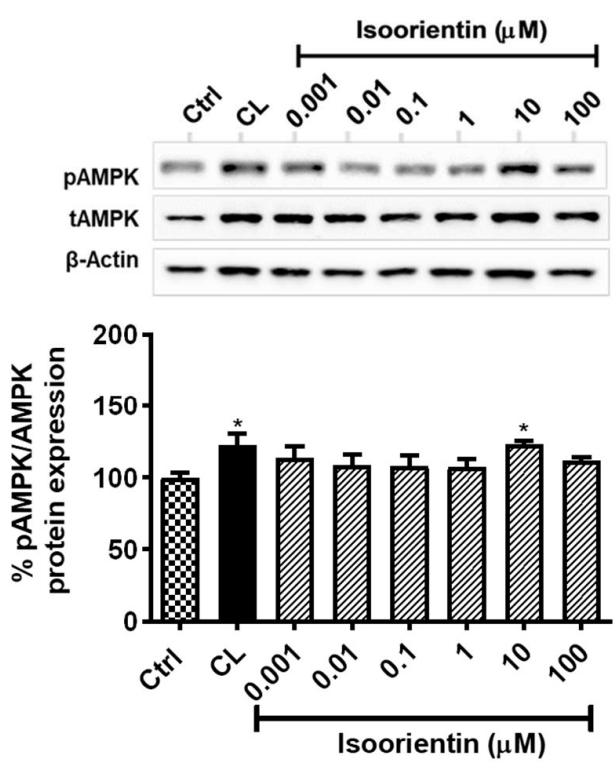

(b)

Figure 4. Isoorientin increased protein kinase B (pAKT), (a) 5' AMP-activated protein kinase (pAMPK); and $(\mathbf{b})$ protein expression in fully differentiated 3T3-L1 adipocytes. Matured 3T3-L1 adipocytes were treated with or without positive control CL-316,243 (CL), isoproterenol (Isopr) used at $1 \mu \mathrm{M}$ and various doses of isoorientin $(0.001,0.01,0.1,1,10$, and $100 \mu \mathrm{M})$ for 4 hours; thereafter, proteins were extracted for Western blot analysis. Results are expressed as mean \pm SEM of three independent experiments. ${ }^{*} p<0.05,{ }^{* * *} p<0.001$ versus normal control (Ctrl).

\section{Materials and Methods}

\subsection{Materials}

3T3-L1 mouse embryonic fibroblasts (Cat\# CL-173) were purchased from the American Type Culture Collection (Manassas, VA, USA). Dulbecco's modified Eagle's medium (DMEM), Dulbecco's phosphate buffered saline (DPBS), penicillin/streptomycin and trypsin/versene were bought from Lonza Biowhitaker (Walkersville, MD, USA). Fetal bovine serum (FBS) were obtained from Gibco, Invitrogen (EU Approved, origin: South America). Isoorientin ( $\geq 98.0 \%$ purity), CL316243 (CL), dexamethasone (DEX), 3-isobutyl-1-methylxanthine (IBMX), insulin, oil red O (ORO), dimethyl sulfoxide (DMSO), Sodium biocarbonate $\left(\mathrm{NaHCO}_{3}\right)$, as well as phenol red and glucose-free DMEM were procured from Sigma-Aldrich (St. Louis, MO, USA). The ViaLight ${ }^{\mathrm{TM}}$ plus ATP kit was obtained from Lonza (Basel, Switzerland). Bradford and RC DC protein assay kits were bought from Bio-Rad Laboratories (Hercules, CA, USA). Protease and phosphatase inhibitor tablets were purchased from Roche (South San Francisco, CA, USA). Seahorse XF96 microplate plates, Seahorse XF Assay media, and Seahorse XF-Cell Mito Stress assay kits were purchased from Agilent (Santa Clare, CA, USA). Cell Signalling Technology (Beverly, MA, USA) supplied primary antibodies including AKT (cat\# 9272S), p-AKT (Ser473) (cat\# 9271S), AMPK (cat\# 2532), p-AMPK (Thr172) (cat\# 2535S). The housekeeping $\beta$-actin (cat\# sc-47778), secondary antibodies goat anti-rabbit (cat\# sc-2004), and IgG- horseradish peroxidase were purchased from Santa Cruz Biotechnology (Dallas, Texas, USA). All other reagents and chemical were from Sigma unless specified.

\subsection{Methods}

\subsubsection{Cell Culture and Differentiation of 3T3-L1 Adipocytes}

Mouse 3T3-L1 pre-adipocytes were cultured in a growth medium (DMEM containing 10\% FBS), in a humidified atmosphere of $37^{\circ} \mathrm{C}$ and $5 \% \mathrm{CO}_{2}$, as previously described [21]. Briefly, pre-adipocytes 
were seeded in a growth media at a density of 20,000 cells $/ \mathrm{mL}$, which allowed them to reach confluency within 3 days in 24-well plates for ORO staining, 96-well microplates for ATP and MTT assays, as well as 6-well plates for protein expression analysis. Briefly, the differentiation process was as follows: upon confluency (day 0), growth medium was substituted with adipocyte differentiation media (DMEM containing 10\% FBS, $0.5 \mathrm{mM}$ IBMX, $1 \mu \mathrm{M}$ DEX, and $1 \mu \mathrm{g} / \mathrm{mL}$ insulin), for 2 days. On day 3 , the medium was changed to insulin media or adipocyte maintenance media (DMEM containing $1 \mu \mathrm{g} / \mathrm{mL}$ insulin) for a further 2 days. From day 5, cells were maintained in the growth medium, until day 8 . After day 8 , cells were fully differentiated to mature adipocytes; thereafter, relevant assays were performed.

\subsubsection{Preparation of Compounds and Treatment}

Isoorientin stock was prepared by dissolving $10 \mathrm{mg}$ in $100 \%$ DMSO $(1 \mathrm{~mL})$ to prepare a concentrated solution of $22.30 \mathrm{mM}$, which was stored at $-80^{\circ} \mathrm{C}$ until further use. The initial stock was further diluted in media to yield working concentrations of $0.001,0.01,0.1,1,10$, and $100 \mu \mathrm{M}$. All compounds, including isoorientin and two positive controls of pioglitazone and CL-316,243, were prepared by diluting the appropriate amounts of a stock solution in phenol red free DMEM (supplemented with $8 \mathrm{mM}$ glucose, $3.7 \mathrm{~g} / \mathrm{L} \mathrm{NaHCO}_{3}$, and $0.1 \%(w / v)$ bovine serum albumin (BSA)), to yield the final working concentrations. To eliminate interference of DMSO in the bioactivity of compounds, the final DMSO in all experiments was $<0.001 \%$, as previously reported [22]. Briefly, the treatment process involved a nutrient deprivation step where differentiated adipocytes were cultured in phenol red free DMEM, without glucose and serum (supplemented with $3.7 \mathrm{~g} / \mathrm{L} \mathrm{NaHCO}_{3}, 0.1 \%$ (w/v) BSA), for $30 \mathrm{~min}$ prior to the addition of the relevant treatment. Cells were subsequently treated with various concentrations of isoorientin $(0.001,0.01,0.1,1,10$, and $100 \mu \mathrm{M})$ in DMEM without phenol red (containing $8 \mathrm{mM}$ glucose, $3.7 \mathrm{~g} / \mathrm{L} \mathrm{NaHCO} 3,0.1 \%(\mathrm{w} / \mathrm{v}) \mathrm{BSA}$ ), for 4 hours, in a humidified atmosphere of $37^{\circ} \mathrm{C}$ and $5 \% \mathrm{CO}_{2}$. Positive controls such as insulin $(1 \mu \mathrm{M})$, metformin $(1 \mu \mathrm{M})$, isoproterenol $(10 \mu \mathrm{M})$, and CL-316,243 $(10 \mu \mathrm{M})$, were also added for 4 hours [23]. Dose and time selection was based on previously published evidence from our lab, demonstrating that isoorientin is able to improve glucose uptake in $\mathrm{C}_{2} \mathrm{C}_{12}$ cells exposed to isoorientin for $3 \mathrm{~h}$ [24].

\subsubsection{ATP Content and Glucose Uptake Determination}

After treatment for 4 hours, the ATP content, as a measurement of metabolic activity, was quantified using the ViaLight ${ }^{\mathrm{TM}}$ plus sample kit (Lonza, Basel, Switzerland), following the manufacturer's protocol. Luminescence was measured using a BioTek ${ }^{\circledR}$ FLx800 plate reader equipped with Gen $5^{\circledR}$ software (BioTek Instruments Inc., Winooski, VT, USA). Glucose uptake was performed as previously described by [25] with slight modifications. Briefly, after 4 hours of treatment, adipocytes were incubated with DMEM without phenol red, containing $0.5 \mu \mathrm{Ci} / \mathrm{mL}$ 2-deoxy- $\left[{ }^{3} \mathrm{H}\right]$-D-glucose (Radiolabeled Chemicals, St Louis, MO, USA), for $15 \mathrm{~min}$ at $37^{\circ} \mathrm{C}$ in $5 \% \mathrm{CO}_{2}$. Subsequently, 2-deoxy-[ $\left.{ }^{3} \mathrm{H}\right]$-D-glucose uptake was assessed by liquid scintillation, as described by [24]. Thereafter, 2-deoxy-[ $\left.{ }^{3} \mathrm{H}\right]-\mathrm{D}-$ glucose uptake was assessed in the lysate by liquid scintillation (2220 CA, Packard Tri-Carbseries, PerkinElmer, Downers Crove, IL, USA).

\subsubsection{Intracellular Lipid Content and Glycerol Determination}

Intracellular lipid accumulation was determined using ORO, as described by [16]. After 4 hours of treatment, media was collected for glycerol, cells were stained with $0.7 \%(v / v) \mathrm{ORO}$, and lipid droplets were visualized using a Nikon inverted fluorescent microscope (Nikon eclipse Ti; Nikon, Japan), equipped with a camera (Nikon DXM1200) and NIS-Element imaging software. Stained droplets were dissolved with isopropanol and quantified by spectrophotometric analysis at $570 \mathrm{~nm}$ using a BioTek ${ }^{\circledR}$ ELx800 plate reader equipped with Gen $5^{\circledR}$ software for data acquisition. The lipid content was normalized to cell density (crystal violet staining measured at $570 \mathrm{~nm}$ ). Moreover, the amount of free glycerol in the collected cell culture medium was ascertained using the fluorescent Glycerol 
Release Assay kit from Bio vision Inc. (Milpitas, CA, USA), according to the manufacturer's protocol. Free glycerol content was normalized to cell density (crystal violet measured at $570 \mathrm{~nm}$ ).

\subsubsection{Assessing Mitochondrial Bioenergetics}

Mitochondrial bioenergetics were assessed by determining OCR, using a Mito Stress assay kit and XF96 Extracellular Flux analyzer (Agilent, Santa Clara, CA, USA), as described previously [26], with some modifications. Briefly, 3T3-L1 adipocytes were seeded in 96-well XF microplates at 10,000 cells per well, which reached confluence after 24 hours. Subsequently, cells were differentiated and treated as previously described in the cell differentiation and treatment section; however, the Seahorse assay was performed on day 5 of differentiation. Briefly, after treatment, cells were washed twice with $180 \mu \mathrm{L}$ of pre-warmed XF assay medium (XF base medium containing $8 \mathrm{mM}$ glucose, $2 \mathrm{mM}$ glutamine, and $1 \mathrm{mM}$ sodium pyruvate at $\mathrm{pH} 7.4$ ) and incubated with the same volume of $\mathrm{XF}$ assay medium at $37^{\circ} \mathrm{C}$ in a non- $\mathrm{CO}_{2}$ incubator for $1 \mathrm{~h}$, to equilibrate temperature and $\mathrm{pH}$ prior to OCR measurement. ATP production and maximal respiration were determined by treating the cells with $1 \mu \mathrm{M}$ oligomycin and $0.75 \mu \mathrm{M}$ carbonyl cyanide-4-trifluoromethoxy-phenylhydrazone (FCCP). After the assay, the Bradford protein assay was used to determine protein concentration, and then OCR ( $\mathrm{pmol} / \mathrm{min}$ ) was normalized relative to the protein content. OCR was reported as absolute rates, i.e., $\mathrm{pmol} / \mathrm{min} / \mathrm{mg}$.

\subsubsection{Western Blot Analysis}

Western blot analysis was performed as described by [25] with some modifications. In brief, the primary antibodies AMPK (1:1000), p-AMPK (Thr172) (1:800), AKT (1:1000), p-AKT (Ser473) (1:1000), and the reference protein $\beta$-actin (1:1000) were used and incubated at $4{ }^{\circ} \mathrm{C}$ overnight. The following day, the respective HRP-conjugated secondary antibodies goat anti-mouse or goat anti-rabbit (1:4000), were applied for $90 \mathrm{~min}$ at room temperature. Protein bands were visualized using the ChemiDoc ${ }^{\mathrm{TM}} \mathrm{MP}$ system (BioRad, Cressier, Switzerland), and Image Lab software version 5.2.1 was used for analysis.

\subsubsection{Statistical Analysis}

All results are expressed as the means \pm SEM of three independent biological experiments. The significance of difference was determined using one-way analysis of variance (ANOVA), followed by Tukey's post hoc test using GraphPad Prism version 6.07 (GraphPad Software Inc., San Diego, CA, USA). Results were considered significant at $p<0.05$.

\section{Discussion}

Murine 3T3-L1 adipocytes remain a well-established and suitable model for investigating adipocytic lipid metabolism and obesity in vitro. This is evidenced by an increasing number of studies that utilize this model to screen for anti-obesity properties of plant extracts and their natural products [14,27]. During the differentiation of 3T3-L1 pre-adipocytes, the changes in cell size and number are known to be regulated by adipogenic transcription factors, including CCAAT/enhancer binding protein (C/EBPs) and PPAR $\gamma$ [28]. These transcription factors are known to promote adipogenesis in various cell models of obesity, as reviewed elsewhere [29]. Furthermore, AKT and AMPK signaling pathways are also known to play a crucial role in the regulation of adipogenesis. In fact, the activation of the AKT pathway promotes adipogenesis by upregulating $\operatorname{PPAR} \gamma$ and $\mathrm{C} / \mathrm{EBP} \alpha$, hence increasing lipid accumulation in 3T3-L1 adipocytes [30]. Activation of AKT can also promote insulin-dependent glucose uptake by mainly promoting translocation of glucose transporters, as observed in skeletal muscles exposed to palmitate [31]. On the other hand, activation of the AMPK pathway, a key sensor and regulator of cellular energy, inhibits adipogenesis, in part by blocking the expression of PPAR $\gamma$ in experimental models of obesity [32,33]. AMPK is also implicated in insulin-independent glucose uptake, and this process is revised in detail by Hayley et al. [34], which looks at various experimental models of insulin resistance. Importantly, as an integral process in controlling the process 
of adipogenesis, these pathways are known to greatly impact mitochondrial function in conditions of obesity. Consistently, activation of AMPK has been associated with increased $\beta$-oxidation, including the process of mitochondrial energetics [26]. Therefore, the treatments that reduce lipid accumulation, while enhancing energy metabolism via the aforementioned mechanisms, may provide an effective preventive or therapeutic approach for obesity.

The current study reports on essential evidence on the impact of isoorientin on lipid accumulation in an in vitro model of obesity. Such evidence includes, the observed concentration-dependent beneficial effects of isoorientin on blocking lipid storage, enhancing glycerol release, as well as improving metabolic activity and mitochondrial function [35]. Although the observed beneficial effects of isoorientin on these cultured 3T3-L1 adipocytes were concentration-independent, it was clear that doses between 1 and $10 \mu \mathrm{M}$ presented with enhanced effects compared to the untreated control, and their activity was comparable to tested positive controls of CL, isoproterenol and metformin. Interestingly, the beneficial effects reported with the conducted bioassays of lipid accumulation and mitochondrial respiration showed consistent results in activating some of the crucial pathways involved in the regulation of these processes, especially isoorientin doses $\geq 1 \mu \mathrm{M}$. For instance, higher doses of isoorientin significantly enhanced the expression of AKT and AMPK, which could have inhibited lipogenesis by increasing energy expenditure. These results also link well with the implication of AMPK in the regulation of mitochondrial energetics and oxygen consumption in 3T3-L1 adipocytes, as previously reported [35]. Although other studies, or rather findings using plants containing this flavone, have been investigated for their anti-obesity properties [6,36], there is generally limited knowledge on the impact of isoorientin on lipid accumulation in matured adipocytes, especially its role in mitochondrial function and regulatory mechanisms.

Furthermore, results from the current study also point to the importance of dose-dependent considerations when investigating the bioactivity of diverse phytochemical compounds, such as isoorientin. Certainly, other researchers [37] have demonstrated that, depending on time, some polyphenols are more stable in human plasma than in cell culture conditions, which may be correlated to improved polyphenol-protein interactions in vivo. Thus, more research targeting the impact of dose and time response of isoorientin in vivo is necessary to better understand its pharmacokinetic profile. This could also highlight the limited investigations that have assessed the bioactivity properties of isoorientin against metabolic complications using in vivo models. Nevertheless, plant extracts containing this flavone have been shown to have an enhanced effect in lowering blood glucose levels, in protecting against $\beta$-cell damage, and improving the antioxidant status of diabetic rats [38,39]. Consistently, others show that plants, such as buckwheat containing this flavone, can ameliorate the insulin resistance and improve energy and glucose metabolism in fructose-diet exposed or estrogen-deficient rats [40,41]. Thus, these findings further highlight the potential and important role that isoorientin can play in energy regulation in conditions of enhanced lipid accumulation. However, additional studies are required to assess the impact of this dietary flavone on key adipocyte browning specific markers, such as peroxisome proliferator activated receptor-alpha $(\operatorname{PPAR} \alpha)$, peroxisome proliferator-activated receptor gamma coactivator $1 \alpha(\mathrm{PGC} 1 \alpha)$, and sirtuin (SIRT1), using established in vivo models.

\section{Conclusions}

This study advocates the anti-obesity effect of isoorientin and describes some of the underlying mechanisms involved in its observed bioactivity in fully differentiated 3T3-L1 adipocytes. It also provides the first evidence that isoorientin could enhance mitochondrial respiration by stimulating ATP production and the oxygen consumption rate, an important process linked with efficient mitochondrial function. However, further investigations on the expression of key adipocyte browning specific markers, are still required to provide a better understanding of the underlying molecular mechanisms. Consistently, in vivo studies exploring dose and time response to isoorientin treatment, are needed to improve our understanding of its pharmacokinetic profile. 
Author Contributions: K.Z., S.E.M.-M., C.J.F.M., and A.P.K. were in charge of study conception and design. K.Z., S.X.H.M., N.O., and S.E.M-M. were responsible for data acquisition and analysis. K.Z., P.V.D., S.S., P.O., and S.E.M.-M. wrote the manuscript. J.L. and L.T. edited the manuscript. All authors read and approved the final manuscript.

Funding: This work was funded by the National Research Foundation (NRF) Thuthuka Programme grant 80618 and 113674 (to SE Mazibuko-Mbeje). Baseline funding from Biomedical Research and Innovation Platform of the South African Medical Research Council (SAMRC) and University of Zululand is also acknowledged. The authors also acknowledge financial support for K. Ziqubu, as a research intern on the SAMRC Research Capacity Development (RCD) program. Similarly, the researcher PV Dludla was partially supported as a Post-Doctoral Fellow by funding from the SAMRC through its division of Research Capacity Development under the Intra-Mural Postdoctoral Fellowship Programme from funding received from the South African Treasury. The content hereof is the sole responsibility of the authors and do not necessary represent the official views of the SAMRC or the funders.

Conflicts of Interest: The authors declare no conflict of interest

\section{References}

1. Patti, A.M.; Al-Rasadi, K.; Giglio, R.V.; Nikolic, D.; Mannina, C.; Castellino, G.; Chianetta, R.; Banach, M.; Cicero, A.F.G.; Lippi, G.; et al. Natural approaches in metabolic syndrome management. Arch. Med. Sci. AMS 2018, 14, 422-441. [CrossRef]

2. Fu, C.; Jiang, Y.; Guo, J.; Su, Z. Natural Products with Anti-Obesity Effects and Different Mechanisms of Action. J. Agric. Food Chem. 2016, 64, 9571-9585. [CrossRef]

3. Thounaojam, M.C.; Nammi, S.; Jadeja, R. Natural products for the treatment of obesity, metabolic syndrome, and type 2 diabetes. Evid.-Based Complement. Altern. Med. eCAM 2013, 2013, 871018. [CrossRef]

4. Dludla, P.V.; Nkambule, B.B.; Jack, B.; Mkandla, Z.; Mutize, T.; Silvestri, S.; Orlando, P.; Tiano, L.; Louw, J.; Mazibuko-Mbeje, S.E. Inflammation and Oxidative Stress in an Obese State and the Protective Effects of Gallic Acid. Nutrients 2018, 11, 23. [CrossRef]

5. Alonso-Castro, A.J.; Zapata-Bustos, R.; Gomez-Espinoza, G.; Salazar-Olivo, L.A. Isoorientin reverts TNF-alpha-induced insulin resistance in adipocytes activating the insulin signaling pathway. Endocrinology 2012, 153, 5222-5230. [CrossRef]

6. Poudel, B.; Nepali, S.; Xin, M.; Ki, H.H.; Kim, Y.H.; Kim, D.K.; Lee, Y.M. Flavonoids from Triticum aestivum inhibit adipogenesis in 3T3-L1 cells by upregulating the insig pathway. Mol. Med. Rep. 2015, 12, 3139-3145. [CrossRef]

7. Koeppen, B.H.; Smit, J.B.; Roux, D.G. The flavone C-glycosides and flavonol O-glycosides of Aspalathus acuminatus (rooibos tea). Biochem. J. 1962, 83, 507-511. [CrossRef]

8. Joubert, E.; de Beer, D.; Malherbe, C.J.; Muller, N.; Bonnet, S.L.; van der Westhuizen, J.H.; Ferreira, D. Occurrence and sensory perception of Z-2-(beta-d-glucopyranosyloxy)-3-phenylpropenoic acid in rooibos (Aspalathus linearis). Food Chem. 2013, 136, 1078-1085. [CrossRef]

9. Sezik, E.; Aslan, M.; Yesilada, E.; Ito, S. Hypoglycaemic activity of Gentiana olivieri and isolation of the active constituent through bioassay-directed fractionation techniques. Life Sci. 2005, 76, 1223-1238. [CrossRef]

10. Aragao, D.M.; Guarize, L.; Lanini, J.; da Costa, J.C.; Garcia, R.M.; Scio, E. Hypoglycemic effects of Cecropia pachystachya in normal and alloxan-induced diabetic rats. J. Ethnopharmacol. 2010, 128, 629-633. [CrossRef]

11. Im, J.Y.; Ki, H.H.; Xin, M.; Kwon, S.U.; Kim, Y.H.; Kim, D.K.; Hong, S.P.; Jin, J.S.; Lee, Y.M. Anti-obesity effect of Triticum aestivum sprout extract in high-fat-diet-induced obese mice. Biosci. Biotechnol. Biochem. 2015, 79, 1133-1140. [CrossRef]

12. Dludla, P.V.; Muller, C.J.; Louw, J.; Joubert, E.; Salie, R.; Opoku, A.R.; Johnson, R. The cardioprotective effect of an aqueous extract of fermented rooibos (Aspalathus linearis) on cultured cardiomyocytes derived from diabetic rats. Phytomed. Int. J. Phytother. Phytopharmacol. 2014, 21, 595-601. [CrossRef]

13. Mazibuko, S.E.; Muller, C.J.; Joubert, E.; de Beer, D.; Johnson, R.; Opoku, A.R.; Louw, J. Amelioration of palmitate-induced insulin resistance in $\mathrm{C}(2) \mathrm{C}(1)(2)$ muscle cells by rooibos (Aspalathus linearis). Phytomed. Int. J. Phytother. Phytopharmacol. 2013, 20, 813-819.

14. Sanderson, M.; Mazibuko, S.E.; Joubert, E.; de Beer, D.; Johnson, R.; Pheiffer, C.; Louw, J.; Muller, C.J. Effects of fermented rooibos (Aspalathus linearis) on adipocyte differentiation. Phytomed. Int. J. Phytother. Phytopharmacol. 2014, 21, 109-117. [CrossRef]

15. Kakkar, A.K.; Dahiya, N. Drug treatment of obesity: Current status and future prospects. Eur. J. Intern. Med. 2015, 26, 89-94. [CrossRef] 
16. Kupeli, E.; Aslan, M.; Gurbuz, I.; Yesilada, E. Evaluation of in vivo biological activity profile of isoorientin. Zeitschrift fur Naturforschung C 2004, 59, 787-790. [CrossRef]

17. Lim, J.H.; Park, H.S.; Choi, J.K.; Lee, I.S.; Choi, H.J. Isoorientin induces Nrf2 pathway-driven antioxidant response through phosphatidylinositol 3-kinase signaling. Arch. Pharmacal Res. 2007, 30, 1590-1598. [CrossRef]

18. Yuan, L.; Han, X.; Li, W.; Ren, D.; Yang, X. Isoorientin Prevents Hyperlipidemia and Liver Injury by Regulating Lipid Metabolism, Antioxidant Capability, and Inflammatory Cytokine Release in High-Fructose-Fed Mice. J. Agric. Food Chem. 2016, 64, 2682-2689. [CrossRef]

19. Yuan, L.; Wang, J.; Wu, W.; Liu, Q.; Liu, X. Effect of isoorientin on intracellular antioxidant defence mechanisms in hepatoma and liver cell lines. Biomed. Pharmacother. 2016, 81, 356-362. [CrossRef]

20. Anilkumar, K.; Reddy, G.V.; Azad, R.; Yarla, N.S.; Dharmapuri, G.; Srivastava, A.; Kamal, M.A.; Pallu, R. Evaluation of Anti-Inflammatory Properties of Isoorientin Isolated from Tubers of Pueraria tuberosa. Oxid. Med. Cell. Longev. 2017, 2017, 5498054. [CrossRef]

21. Mazibuko, S.E.; Joubert, E.; Johnson, R.; Louw, J.; Opoku, A.R.; Muller, C.J. Aspalathin improves glucose and lipid metabolism in 3T3-L1 adipocytes exposed to palmitate. Mol. Nutr. Food Res. 2015, 59, 2199-2208. [CrossRef]

22. Dludla, P.V.; Jack, B.; Viraragavan, A.; Pheiffer, C.; Johnson, R.; Louw, J.; Muller, C.J. A dose-dependent effect of dimethyl sulfoxide on lipid content, cell viability and oxidative stress in 3T3-L1 adipocytes. Toxicol. Rep. 2018, 5, 1014-1020. [CrossRef]

23. Asano, H.; Kanamori, Y.; Higurashi, S.; Nara, T.; Kato, K.; Matsui, T.; Funaba, M. Induction of beige-like adipocytes in 3T3-L1 cells. J. Vet. Med. Sci. 2014, 76, 57-64. [CrossRef]

24. Mazibuko, S. In Vitro and In Vivo Effect of Aspalathu linearis and Its Major Polyphenols on Carbohydrate and Lipid Metabolism in Insulin Resistant Models; University of Zululand: Richards Bay, South Africa, 2014.

25. Johnson, R.; Dludla, P.; Joubert, E.; February, F.; Mazibuko, S.; Ghoor, S.; Muller, C.; Louw, J. Aspalathin, a dihydrochalcone $\mathrm{C}$-glucoside, protects $\mathrm{H} 9 \mathrm{c} 2$ cardiomyocytes against high glucose induced shifts in substrate preference and apoptosis. Mol. Nutr. Food Res. 2016, 60, 922-934. [CrossRef]

26. Mazibuko-Mbeje, S.E.; Dludla, P.V.; Johnson, R.; Joubert, E.; Louw, J.; Ziqubu, K.; Tiano, L.; Silvestri, S.; Orlando, P.; Opoku, A.R.; et al. Aspalathin, a natural product with the potential to reverse hepatic insulin resistance by improving energy metabolism and mitochondrial respiration. PLOS ONE 2019, 14, e0216172. [CrossRef]

27. Karri, S.; Sharma, S.; Hatware, K.; Patil, K. Natural anti-obesity agents and their therapeutic role in management of obesity: A future trend perspective. Biomed. Pharmacother. 2019, 110, 224-238. [CrossRef]

28. Valli, V.; Heilmann, K.; Danesi, F.; Bordoni, A.; Gerhauser, C. Modulation of Adipocyte Differentiation and Proadipogenic Gene Expression by Sulforaphane, Genistein, and Docosahexaenoic Acid as a First Step to Counteract Obesity. Oxid. Med. Cell. Longev. 2018, 2018, 1617202. [CrossRef]

29. Ruiz-Ojeda, F.J.; Ruperez, A.I.; Gomez-Llorente, C.; Gil, A.; Aguilera, C.M. Cell Models and Their Application for Studying Adipogenic Differentiation in Relation to Obesity: A Review. Int. J. Mol. Sci. 2016, 17, 1040. [CrossRef]

30. Chen, Y.Y.; Lee, M.H.; Hsu, C.C.; Wei, C.L.; Tsai, Y.C. Methyl cinnamate inhibits adipocyte differentiation via activation of the CaMKK2-AMPK pathway in 3T3-L1 preadipocytes. J. Agric. Food Chem. 2012, 60, 955-963. [CrossRef]

31. Zhang, Z.; Liu, H.; Liu, J. Akt activation: A potential strategy to ameliorate insulin resistance. Diabetes Res. Clin. Pract. 2019, 156, 107092. [CrossRef]

32. Huang, B.; Yuan, H.D.; Kim, D.Y.; Quan, H.Y.; Chung, S.H. Cinnamaldehyde prevents adipocyte differentiation and adipogenesis via regulation of peroxisome proliferator-activated receptor-gamma (PPARgamma) and AMP-activated protein kinase (AMPK) pathways. J. Agric. Food Chem. 2011, 59, 3666-3673. [CrossRef]

33. Mazibuko-Mbeje, S.E.; Dludla, P.V.; Roux, C.; Johnson, R.; Ghoor, S.; Joubert, E.; Louw, J.; Opoku, A.R.; Muller, C.J. Aspalathin-Enriched Green Rooibos Extract Reduces Hepatic Insulin Resistance by Modulating PI3K/AKT and AMPK Pathways. Int. J. Mol. Sci. 2019, 20, 633. [CrossRef]

34. O’Neill, H.M. AMPK and Exercise: Glucose Uptake and Insulin Sensitivity. Diabetes Metab. J. 2013, 37, 1-21. [CrossRef]

35. Liu, M.; Zheng, M.; Cai, D.; Xie, J.; Jin, Z.; Liu, H.; Liu, J. Zeaxanthin promotes mitochondrial biogenesis and adipocyte browning via AMPKalpha1 activation. Food Funct. 2019, 10, 2221-2233. [CrossRef] 
36. Luan, G.; Wang, Y.; Wang, Z.; Zhou, W.; Hu, N.; Li, G.; Wang, H. Flavonoid Glycosides from Fenugreek Seeds Regulate Glycolipid Metabolism by Improving Mitochondrial Function in 3T3-L1 Adipocytes in Vitro. J. Agric. Food Chem. 2018, 66, 3169-3178. [CrossRef]

37. Xiao, J.; Hogger, P. Stability of dietary polyphenols under the cell culture conditions: Avoiding erroneous conclusions. J. Agric. Food Chem. 2015, 63, 1547-1557. [CrossRef]

38. Malik, A.; Jamil, U.; Butt, T.T.; Waquar, S.; Gan, S.H.; Shafique, H.; Jafar, T.H. In silico and in vitro studies of lupeol and iso-orientin as potential antidiabetic agents in a rat model. Drug Des. Dev. Ther. 2019, 13, 1501-1513. [CrossRef]

39. Xu, G.K.; Qin, X.Y.; Wang, G.K.; Xie, G.Y.; Li, X.S.; Sun, C.Y.; Liu, B.L.; Qin, M.J. Antihyperglycemic, antihyperlipidemic and antioxidant effects of standard ethanol extract of Bombax ceiba leaves in high-fat-dietand streptozotocin-induced Type 2 diabetic rats. Chin. J. Nat. Med. 2017, 15, 168-177. [CrossRef]

40. Goss, M.J.; Nunes, M.L.O.; Machado, I.D.; Merlin, L.; Macedo, N.B.; Silva, A.M.O.; Bresolin, T.M.B.; Santin, J.R. Peel flour of Passiflora edulis Var. Flavicarpa supplementation prevents the insulin resistance and hepatic steatosis induced by low-fructose-diet in young rats. Biomed. Pharmacother. 2018, 102, 848-854. [CrossRef]

41. Yang, H.J.; Lim, J.H.; Park, K.J.; Kang, S.; Kim, D.S.; Park, S. Methyl jasmolate treated buckwheat sprout powder enhances glucose metabolism by potentiating hepatic insulin signaling in estrogen-deficient rats. Nutrition 2016, 32, 129-137. [CrossRef]

Sample Availability: Samples of the compounds are available through commercial sources. 